\title{
Doença renal crônica e doenças periodontais
}

\section{Chronic Renal Disease and Periodontal Diseases}

\section{RESUMO}

Introdução: Doenças periodontais (DPs) são doenças multifatoriais, polimicrobianas, infecto-inflamatórias que acometem os tecidos periodontais de sustentação e de suporte dos dentes. Ocorre resposta inflamatória e imunológica em decorrência, principalmente do acúmulo de biofilme dentário. Comumente encontram-se nos indivíduos doentes gengivite e periodontite. Doença renal crônica (DRC) trata-se de patologia sistêmica que ocasiona perda irreversível da função renal. Os fatores etiológicos que levam a instalação da DRC são comumente a diabetes mellitus, a hipertensão arterial, nefropatias túbulo-intersticiais, processos renais obstrutivos crônicos e glomerulonefrites. Objetivo: O objetivo deste artigo foi evidenciar como a DRC pode influir no transcurso das DP e como as DP podem alterar o andamento da DRC. Metodologia: Realizou-se revisão bibliográfica com busca nas bases bibliográficas: PubMED, LILACS, Google Acadêmico de estudos e artigos acerca da presença das duas doenças, DPs e DRC, convivendo concomitantemente nos pacientes. Resultados: Em âmbito odontológico deve haver manutenção preventiva de estado de saúde bucal satisfatório em pacientes com DRC. DRC pode agir como fator de risco para instalação de DPs. Por outro lado a presença de periodontite pode agir como fator de risco para ocorrência da DRC. A inflamação proveniente das DPs pode propiciar evolução desfavorável de doenças sistêmicas, como a DRC. Conclusão: $\mathrm{O}$ conhecimento e conscientização pelo cirurgião dentista acerca das manifestações bucais ocasionadas pela DRC, bem como acerca das complicações sistêmicas que podem ocorrer em decorrência de problemas odontológicos, tais como as DPs é fundamental para que o mesmo possa abordar corretamente os pacientes portadores da doença durante os procedimentos odontológicos.

Palavras-chave: Inflamação. Doenças Periodontais. Periodontite. Higiene Bucal. Insuficiência Renal Crônica.

\begin{abstract}
Introduction: Periodontal diseases (PDs) are multifactorial, polymicrobial, infectiousinflammatory diseases that affect the periodontal tissues of support and support of the teeth. Inflammatory and immunological response occurs mainly due to the accumulation of dental biofilm. Gingivitis and periodontitis are commonly found in individuals. Chronic kidney disease (CKD) is systemic pathology that causes irreversible loss of renal function. Etiological factors that lead to establishment of CKD are commonly diabetes mellitus, arterial hypertension, tubulo-interstitial nephropathies, chronic obstructive renal processes and glomerulonephritis. Objective: The objective of this article was to show how CKD can influence the course of PD and how PD can change the progression of CKD. Methodology: Bibliographic review was carried out with a search of the bibliographic bases: PubMED, LILACS, Google Academic of studies and articles on the presence of two diseases, PD and CKD, coexisting concomitantly in patients. Results: In dental field there should be preventive maintenance of a satisfactory oral health status in patients with CKD. DRC can act as a risk factor for the installation of DPs. On the other hand, the presence of periodontitis may act as a risk factor for the occurrence of CKD. Inflammation from PDs may lead to unfavorable evolution of systemic diseases, such as CKD. Conclusion: The knowledge and awareness by the dental surgeon about the oral manifestations caused by CKD, as well as about the systemic complications that can occur due to dental problems, such as PDs, is fundamental that it can correctly approach the patients with the disease during dental procedures.
\end{abstract}

Keywords: Inflammation. Periodontal Diseases. Periodontitis. Oral Hygiene. Renal Insufficiency, Chronic.

\section{Sérgio Spezzia ${ }^{1}$}

${ }^{1}$ Cirurgião Dentista. Especialista em Saúde da Mulher no Climatério pela Faculdade de Saúde Pública da Universidade de São Paulo. Especialista em Gestão Pública pela Universidade Tecnológica Federal do Paraná. Especialista em Gestão em Saúde pela Universidade Federal de São Paulo. Especialista em Adolescência para Equipe Multidisciplinar e Mestre em Ciências pela Escola Paulista de Medicina - Universidade Federal de São Paulo.

\section{Endereço para Correspondência:} Sérgio Spezzia

Rua Silva Bueno, 1001, Ipiranga, CEP 04208-

050, São Paulo, SP

sergio.spezzia@unifesp.br

Submetido em: 19-9-2020

Aceito em: 7-5-2021 


\section{INTRODUÇÃO}

Doenças periodontais (DPs) são doenças multifatoriais, polimicrobianas e infecto-inflamatórias que acometem os tecidos periodontais de sustentação e de suporte dos dentes. Ocorre resposta inflamatória e imunológica em decorrência, principalmente do acúmulo de biofilme dentário. Comumente encontram-se nos indivíduos doentes gengivite e periodontite com maior prevalência. Essas doenças são consideradas um problema de Saúde Pública que é capaz de ocasionar impacto, uma vez que grande parcela da população mundial encontra-se afligida ${ }^{1,2}$.

A DRC também configura um problema de Saúde Pública apresentando morbidade e mortalidade em decorrência. Trata-se de patologia sistêmica que ocasiona perda da função renal com caráter irreversível. Os fatores etiológicos que levam a instalação da DRC são comumente a diabetes mellitus, a hipertensão arterial, nefropatias túbulo-intersticiais, processos renais obstrutivos crônicos e as glomerulonefrites. A obtenção de um diagnóstico de forma precoce da DRC mostra-se dificultosa e sua identificação normalmente pode ocorrer somente na fase avançada, onde existe síndrome urêmica. Recursos para tratamento como o transplante renal, a hemodiálise e a diálise peritoneal mostram-se satisfatórios ${ }^{3}$.

Nesse contexto, sabe-se que algumas patologias sistêmicas crônicas, como a DRC são promotoras de repercussões em âmbito imunológico e podem afligir os pacientes, ocasionando exacerbação inflamatória frente à ação do biofilme bacteriano, acarretando DPs ${ }^{4,5}$.

Relacionado a interação que pode haver por manifestações das duas doenças atuando concomitantemente, têm-se que ambas podem possuir fatores de risco considerados comuns, como é o caso da presença do diabetes mellitus que age causando influência em ambas patologias, além da idade apresentada pelos pacientes e do hábito de fumar, que também podem agir como fatores de risco ${ }^{5-7}$.

A correlação entre DPs e DRC ocorre quando a DRC puder influir desfavoravelmente no quadro das DPs e quando as DPs puderem agir na DRC, produzindo situação em que o processo inflamatório torne-se exacerbado pela presença de ambas patologias ${ }^{8-11}$.

O objetivo deste artigo foi o de evidenciar como a DRC pode influir no transcurso das DP e como as DP podem alterar o andamento da DRC.

\section{MATERIAL E MÉTODOS}

Realizou-se revisão bibliográfica com busca nas bases bibliográficas: PubMED, Literatura Latino-americana e do Caribe em Ciências da Saúde (LILACS), Google Acadêmico de estudos e artigos acerca da presença das duas doenças, DP e DRC, convivendo concomitantemente nos pacientes. Na base PubMED empregou-se a expressão de busca: chronic renal disease and periodontal diseases and periodontitis and dentistry e encontrou-se 79 resultados. No Google Acadêmico empregou-se a expressão: doenças periodontais and periodontite and doença renal crônica and odontologia and 2018 and 2017 and 2016 and 2015 and 2014 e obteve-se aproximadamente 191 resultados. No LILACS empregou-se a expressão: doenças periodontais and doença renal crônica e encontrou-se 25 registros.

Incluiu-se artigos publicados em periódicos nacionais e internacionais nos idiomas inglês e português. 
Excluiu-se após realização de leitura seletiva e analítica de todos os trabalhos levantados, artigos que não possuíam conteúdo concernente com a temática de pesquisa ou que tinham somente conteúdo médico.

Apontamentos de livros, trabalhos, monografias, dissertações e teses também foram considerados válidos.

\section{REVISÃO DE LITERATURA}

As DPs constam de doenças inflamatórias oriundas comumente da ação de bactérias gram negativas. Nelas ocorre atuação de periodontopatógenos que desencadeiam a destruição tecidual na região que circunda os elementos dentais em boca. Mecanismos imuno-inflamatórios que visam proteger o hospedeiro da invasão tecidual são ativados, devido a ação dos microrganismos. A atividade imunológica em decorrência promove inflamação local. A permanência continuada de processos reacionais protetores frente às agressões pode desencadear perda óssea periodontal. A evolução de problemas periodontais está relacionada a presença do biofilme dentário e a suscetibilidade do hospedeiro. Sabe-se, nesse contexto, que o biofilme dentário é constituído por comunidades polimicrobianas complexas que situam-se sobre os dentes ${ }^{1,2,12-15}$.

Existem determinados fatores que contribuem com a instalação das DPs, são eles: biofilme dentário; influência oriunda de patologias sistêmicas; estresse; ação de medicamentos que foram prescritos; tabagismo e influências hormonais, que podem exacerbar o aspecto inflamatório das DPs, entre outros ${ }^{16,17}$.

A gengivite consta de alteração patológica de caráter inflamatório reversível que encontra-se limitada aos tecidos marginais onde denota-se edema, eritema, aspecto clínico de sangramento e vermelhidão. Já a periodontite possui caráter de irreversibilidade, promovendo destruição óssea periodontal e bolsas periodontais ${ }^{18,19}$. Na periodontite bactérias gram negativas anaeróbicas atuam, agrupando-se no biofilme dental supra e subgengival. Sabe-se que os microrganismos anaeróbicos gram negativos, como: Porphyromonas gingivalis, Campilobacter rectus, Capnocytophaga sp, Prevotella intermédia são os principais responsáveis por desencadear perda óssea ${ }^{20}$.

A resposta imunológica propiciada por cada qual hospedeiro somada ao potencial microbiano presente irão influir no quadro de periodontite. Na periodontite agem processos imunológicos e inflamatórios que levam a produção de citocinas, prostaglandinas e proteína $C$ reativa. Concomitantemente a reabsorção óssea e a destruição de tecido conjuntivo encontram-se localmente níveis aumentados de inúmeras citocinas, tais como: interleucina (IL) - 1 $\beta$, IL-6 e fator de necrose tumoral (TNF) - alfa ${ }^{21-23}$.

Relacionado a DRC existem determinados fatores de risco que podem levar ao acometimento pela doença, são eles: gênero masculino; raça negra; faixa etária; hábito de fumar; presença de hipertensão arterial sistêmica e diabetes mellitus, dentre outros ${ }^{24}$.

Classifica-se a DRC em estágios, que são configurados em conformidade com o grau de diminuição da filtração glomerular presente. A taxa de filtração glomerular permite avaliar a existência de perda de função renal. O processo inflamatório crônico presente ocasionará modificações quando houver diminuição da função renal ${ }^{25}$.

A imunidade mostra-se comprometida em indivíduos portadores de quadro renal crônico, o que predispõem ao acometimento por infecções mais facilmente. DPs 
e infecções originárias da cavidade bucal agem como foco de infecção e podem gerar complicações sistêmicas nesses pacientes, podendo ocasionar piora no quadro de DRC. $\mathrm{O}$ acúmulo do biofilme dentário nesses indivíduos pode levar mais facilmente a um quadro de periodontite 26,27 .

Pacientes com DRC apresentam algumas manifestações bucais, são elas: DPs; gengivite; periodontite; má oclusão; cárie dentária; mobilidade dentária; erosão dentária; palidez da mucosa bucal; xerostomia; cálculo dentário; hipoplasias de esmalte; perdas dentárias; edentulismo; hálito urêmico; modificações radiográficas dos ossos maxilares e mandibulares; estomatite urêmica; remodelamento ósseo anormal depois do feitio de exodontias; erupção dentária atrasada; hiperplasia gengival e língua geográfica ${ }^{28-30}$.

Em âmbito odontológico deve haver manutenção preventiva de estado de saúde bucal satisfatório em pacientes com DRC. Normalmente, entretanto, depara-se com desconhecimento dos pacientes acerca de que pode haver influência do estado de saúde bucal no seu quadro geral de saúde, além disso nessas circunstâncias ocorre equivocadamente maior cuidado e preocupação com o estado de saúde sistêmica e pouco cuidado com a higienização bucal ${ }^{3,5,11,31}$.

A periodontite detém papel de destaque quando presente em pacientes que possuem concomitantemente DRC, uma vez que pode haver intensificação do processo inflamatório sistêmico e elevação dos níveis de proteína $C$ reativa. Nesse contexto, têm-se que tanto a DRC como a periodontite constam de patologias inflamatórias crônicas, onde os mecanismos inflamatórios de cada qual hospedeiro determinaram o quadro presente ${ }^{32,33}$.

DRC pode agir como fator de risco para instalação de DPs. Por outro lado a presença de periodontite pode agir como fator de risco para ocorrência da DRC. A inflamação proveniente das DPs pode propiciar evolução desfavorável de doenças sistêmicas, como a DRC ${ }^{31,34}$.

Inúmeros estudos afirmam haver uma relação bidirecional entre periodontite e DRC, e sabe-se que uma doença é capaz de influir no quadro da outra, alterando-o ${ }^{11,35-37}$.

A progressão das DPs em portadores de DRC mostra-se aumentada, comparando-se com o que normalmente procede relacionado as DPs em indivíduos não possuidores de $\mathrm{DRC}^{31,34}$.

Em suma, DPs podem afligir o estado de saúde geral dos pacientes e sabe-se que as mesmas podem agir como fator de risco para o aparecimento de determinadas patologias sistêmicas ${ }^{11,38}$. Patógenos e outros produtos oriundos da destruição tecidual ocasionada pelas DPs migram via corrente sanguínea e afligem outros órgãos a nível sistêmico, entre os quais os rins ${ }^{39,40}$.

\section{DISCUSS ÃO}

Na literatura relacionado a pacientes com DRC, averigua-se que existe piora na qualidade de vida apresentada pelos mesmos no decorrer do tratamento para a doença, havendo aumento do prejuízo presente com a necessidade de extensão do período utilizado para o tratamento ${ }^{41}$.

A prevalência de periodontite é maior em pacientes portadores de patologias renais crônicas. Convém frisar que a periodontite, que gera preocupações quando presente 
em portadores de DRC também é capaz de afligir a qualidade de vida desses indivíduos, inclusive sob enfoque psicossocial ${ }^{35}$.

Fatores psicossociais envolvendo a ocorrência de depressão geram higienização bucal precária e acúmulo de biofilme dentário, podendo predispor ao acometimento por DPs. Em estudo que considerou o estado periodontal e a qualidade de vida existente em indivíduos que realizavam hemodiálise, evidenciou-se tal comportamento ${ }^{42}$.

Estudo realizado averiguou como os pacientes que faziam hemodiálise executavam o autocuidado com sua higiene bucal e verificou-se que havia necessidade de empreender orientações acerca dos cuidados essenciais com a higiene bucal por parte do cirurgião dentista, visando melhor qualidade de vida, além de evitar-se complicações correlacionadas com a doença renal presente ${ }^{43}$.

Muitas vezes o quadro da doença em indivíduos que realizam hemodiálise pode piorar, uma vez que esses pacientes costumam eliminar o contato com o cirurgião dentista ou minimizar o número de consultas odontológicas para avaliação do seu estado de saúde bucal ${ }^{44}$.

Consultas odontológicas para avaliação devem ser feitas regularmente pelos pacientes com DRC, visando preventivamente realização de uma higienização bucal correta e eliminar eventuais focos de infecção provenientes da cavidade bucal, no intuito de impedir transtornos no quadro da doença renal.

Conforme estudo realizado existem 57 patologias sistêmicas que podem relacionar-se com as DPs. Nessas patologias e em estudos realizados leva-se em consideração abordagens relacionadas com o processo inflamatório fisiopatológico presente, que podem repercutir em outros locais do organismo, dentre os quais, os tecidos periodontais ${ }^{45}$.

A proteína $C$ reativa constitui um importante indicador na evidenciação de processos inflamatórios, uma vez que configura uma proteína plasmática que encontra-se presente na fase aguda da inflamação ${ }^{46}$.

Convém ressaltar que os níveis de proteína $C$ reativa, que constitui um marcador de processos inflamatórios, aumentam ao passo em que permanece ocorrendo agressão e destruição tecidual. Em contrapartida, inexistindo estímulo agressor, rapidamente procede a redução dos níveis de proteína $C$ reativa ${ }^{46}$.

Caso coexistam patologias inflamatórias num mesmo paciente, pode-se empregar a proteína $C$ reativa para averiguação da provável somatória ou potencialização de graus inflamatórios que pode advir das doenças presentes ${ }^{46}$.

Estudos efetuados evidenciaram que a presença das DPs leva a uma elevação dos níveis de proteína $C$ reativa nos pacientes examinados, sendo possível minimizar esses níveis de proteína $C$ reativa com o feitio da terapia periodontal por intermédio de raspagem e alisamento radicular ${ }^{46}$.

Estudos realizados na atualidade denotam que o tratamento periodontal pode trazer benefícios para a função renal, diminuindo os níveis de marcadores inflamatórios, tais como: IL-6 e proteína C reativa e incrementando a taxa de filtração glomerular ${ }^{47,48}$.

\section{CONCLUSÕES}

O emprego de procedimentos da terapia periodontal pode melhorar a qualidade de vida dos pacientes acometidos por ambas as patologias, uma vez que com esse recurso pode-se minimizar o grau inflamatório presente. 
O paciente portador de DRC deve ser embasado preventivamente pelo cirurgião dentista acerca de como realizar corretamente o autocuidado com sua higienização bucal para que dessa forma seja possível por si próprio obter manutenção do seu estado de saúde periodontal, evitando acúmulo de biofilme, propiciando desfecho satisfatório.

O conhecimento e a conscientização pelo cirurgião dentista acerca das manifestações bucais ocasionadas pela DRC, bem como acerca das complicações sistêmicas que podem ocorrer em decorrência de problemas odontológicos, tais como as DPs é fundamental para que o mesmo possa abordar corretamente os pacientes portadores da doença renal durante os procedimentos odontológicos.

\section{REFERÊNCIAS}

1. 1. Axelsson P, Lindhe J. Effect of controlled oral hygiene procedures on caries and periodontal disease in adults. Results after 6 years. J Clin Periodontol, 1981; 8(3):239-48.

2. 2. Newman MG, Takei HH, Klokkevold PR, Carranza FA. Carranza Periodontia Clínica. 11ª ed. Rio de Janeiro: Elsevier. 2012; p. 370.

3. 3. Cardoso NMM. Fatores Associados à Prevalência de Edentulismo em Pacientes com Doença Renal Crônica: um estudo transversal em Minas Gerais. [Dissertação]. Belo Horizonte: Faculdade de Odontologia - Universidade Federal de Minas Gerais, 2015.

4. 4. Viana HR, Soares CMBM, Tavares M, Teixeira MM, Silva ACS. Inflamação na doença renal crônica: papel de citocinas. J Bras Nefrol, 2011; 33(3):351-64.

5. 5. Costa IA, Guimarães MV. Inter-relação entre a Doença Periodontal e a Doença Renal Crônica. Anais dos Trabalhos da Jornada Odontológica dos Acadêmicos da Católica, 2018, 4(1). ISSN 2448-1726. Disponível em: <http://publicacoesacademicas.unicatolicaquixada.edu.br/index.php/joac/article/view/2492>. Acessado em 24 de fevereiro de 2019.

6. 6. Bokhari SA, Khan AA, Butt AK, Hanif M, Izhar M, Tatakis DN, et al. Periodontitis in coronary heart disease patients: strong association between bleeding on probing and systemic biomarkers. J Clin Periodontol, 2014; 41(11):1048-54.

7. 7. Flores MF, Montenegro MM, Furtado MV, Polanczyk CA, Rosing CK, Haas AN. Periodontal status affects C-reactive protein and lipids in patients with stable heart disease from a tertiary care cardiovascular clinic. J Periodontol, 2014; 85(4):545-53.

8. 8. Chen LP, Hsu SP, Peng YS, Chiang CK, Hung KY. Periodontal disease is associated with metabolic syndrome in hemodialysis patients. Nephrol Dial Transplant, 2011; 26(12):4068-73.

9. 9. Brito F, Almeida S, Figueredo CM, Bregman R, Suassuna JH, Fischer RG. Extent and severity of chronic periodontitis in chronic kidney disease patients. J Periodont Res, 2012; 47(4):426-30.

10. 10. de Souza CM, Braosi AP, Luczyszyn SM, Olandoski M, Kotanko P, Craig RG, et al. Association among oral health parameters, periodontitis, and its treatment and mortality in patients undergoing hemodialysis. J Periodontol, 2014; 85(6):169-78.

11. 11. Sapata DM, Corrêa GO, Anjos Neto-Filho M, Marson FC, Silva CO. Relação entre doença periodontal e doença renal crônica: revisão de literatura. Dental Press Implantol, 2014; 8(1):81-5.

12. 12. Tribble GD, Lamont RJ. Bacterial invasion of epithelial cells and spreading in periodontal tissue. Periodontol 2000, 2010; 52(1):68 - 83.

13. 13. Marsh PD, Devine DA. How is the development of dental biofilms influenced by the host? J Clin Periodontol, 2011; 38(Suppl. 11):28-35.

14. 14. Maçaneiro CAR, Delmonego A, Marín C, Bottan ER. Nível de informação sobre doenças periodontais: relação com o grau de escolaridade. Rev Fac Odontol Lins, 2015; 25(2):11-8.

15. 15. Guardia J, Feron L, Marcon J, Butze JP. Avaliação do nível de conhecimento sobre doenças periodontais dos pacientes em atendimento na clínica de periodontia do centro universitário da serra gaúcha. Braz J Periodontol, 2017; 2(1):23-6.

16. 16. Jeffcoat MK, Lewis CE, Reddy MS, Wang CY, Redford M. Post-menopausal bone loss and its relationship to oral bone loss. Periodontol 2000, 2000; 23(1):94-102.

17. 17. Leite A, Conti R. O tabagismo e a boca. Rev Assoc Paul Cir Dent, 2001; 55:7-14.

18. 18. Engebretson SP, Lalla E, Lamster IB. Periodontitis and systemic disease. NY State Dent J, 1999; 65(8):30-2. 
19. 19. Chiapinotto GA. Etiologia e Prevenção da Doença Periodontal. In: Pinto VG. Saúde Bucal Coletiva. $4^{\mathrm{a}}$. Ed, Ed. Santos: São Paulo; 2000. p. 429-44.

20. 20. Eto FS, Raslan SA, Cortelli JR. Características Microbianas na Saúde e Doença Periodontal. Rev Biociênc Taubaté, 2003; 9(2):45-51.

21. 21. Graves DT, Cochran D. The contribution of interleukin-1 and tumor necrosis factor to periodontal tissue destruction. J Periodontol, 2003; 74(3):391-401.

22. 22. Loos BG. Systemic markers of inflammation in periodontitis. J Periodontol, 2005; 76(11 Suppl):2106-15.

23. 23. Andrukhov O, Ulm C, Reischl H, Nguyen PQ, Matejka M, Rausch-Fan X. Serum cytokine levels in periodontitis patients in relation to the bacterial load. J Periodontol, 2011; 82(6):885-92.

24. 24. Bastos MG, Bregman R, Kirsztajn GM. Chronic kidney diseases: common and harmful, but also preventable and treatable. Rev Assoc Med Bras, 2010; 56(2):248-53.

25. 25. Levey AS, Coresh J, Bolton K, Culleton B, Harvey KS, Ikizler TA et al. K/DOQI clinical practice guidelines for chronic kidney disease: evaluation, classification and stratification. Am J Kidney Dis, 2002; 39:(Suppl 2):S1-S246.

26. 26. Almeida S, Bregman R, Figueredo CM, Fischer RG. Periodontite e doença renal crônica. Rev Hosp Univ Pedro Ernesto, 2013, 12:66-75.

27. 27. Oyetola EO, Owotade FJ, Agbelusi GA, Fatusi AO, Sanusi AA. Oral findings in chronic kidney disease: implications for management in developing countries. BMC Oral Health, 2015; 15:24.

28. 28. Wilczyńska-Borawska M, Malyszko J, Cylwik-Rokicka D, Myśliwiec M. Prosthetic status and treatment needs for lost masticatory function in haemodialysis patients. Arch Med Sci, 2012; 8(1):81-7.

29. 29. Jain S, Singla A, Basavaraj P, Singh S, Singh K, Kundu H. Underlying kidney disease and duration of hemodialysis: an assessment of its effect on oral health. J C Diagn Res, 2014; 8(5):ZC65-ZC69.

30. 30. Palmer SC, Ruospo M, Wong G, Craig JC, Petruzzi M, de Benedittis M et al. Patterns of oral disease in adults with chronic kidney disease treated with hemodialysis. Nephrol Dial Transplant, 2016; 31(10):1647-53.

31. 31. Otávio GMC, Damasceno VMS, Lemos TN. Importância do Conceito de Medicina Periodontal na Integralidade da Assistência à Saúde. Oral Sci, 2014, 6(2):10-7.

32. 32. Ismail FB, Ismail G, Dumitriu AS, Baston C, Berbecar V, Jurubita R et al. Identification of Subgingival Periodontal Pathogens and Association with the Severity of Periodontitis in Patients with Chronic Kidney Diseases: A Cross-Sectional Study . BioMed Research International, 2015; 37(3):1-7.

33. 33. Kim YJ, de Moura LM, Caldas CP, Perozini C, Ruivo GF, Pallos D. Avaliação da condição e risco periodontal em pacientes com doença renal crônica em hemodiálise. Einstein, 2017; 15(2):173-7.

34. 34. Scannapieco FA, Panesar M. Periodontitis and Chronic Kidney Disease. J Periodontol, 2008; 79(9):1617-19.

35. 35. Craig RG, Kotanko P, Kamer AR, Levin NW. Periodontal diseases - a modifiable source of systemic inflammation for the end-stage renal disease patient on haemodialysis therapy? Neph Dial Transpl, 2007; 22(2):312-5.

36. 36. Gonçalves GA, Martins C, Tura LFR, Primo LG. A dimensão educativa da equipe de nefrologia na promoção de saúde bucal de crianças e adolescentes portadores de doença renal crônica. J Bras Nefrol, 2009; 31(3):198-205.

37. 37. Almeida DC, Pereira CS, Granjeiro JM, Machado WAS, Tostes FRV, Barboza ESP. A relação bidirecional entre doença periodontal e doença renal crônica: da progressão da doença renal crônica à terapia renal substitutiva de diálise. Rev Periodontia, 2011; 21(1):73-9.

38. 38. Brasil LO. Medicina periodontal na atualidade [monografia]. Piracicaba: FOP/Unicamp, 2017.

39. 39. Gonçalves EM, Lima DL, de Albuquerque SH, de Carvalho JA, Cariri TFA, de Oliveira CM. Avaliação da perda de inserção dentária em pacientes com doença renal crônica em hemodiálise. J Bras Nefrol, 2011; 33(3):291-4.

40. 40. Vilela EM, Bastos JA, N Ferreira, Chaoubah A, Bastos MG. Treatment of chronic periodontitis decreases serum prohepcidin levels in patients with chronic kidney disease. Clinics, 2011; 66(4):657-62.

41. 41. Guedes KD, Guedes HM. Qualidade de vida do paciente portador de insuficiência renal crônica. Rev Ciênc Saúde, 2012; 5(1):48-53.

42. 42. Guzeldemir E, Toygar HU, Tasdelen B, Torun D. Oral health-related quality of life and periodontal health status in patients undergoing hemodialysis. J Am Dent Assoc, 2009; 140(10):1283-93.

43. 43. Barros DCP, Cordova LHS, Velandia AAL, Souza DM. Avaliação dos hábitos de higiene bucal de pacientes em hemodiálise do Hospital Regional do Vale do Paraíba. Braz J Periodontol, 2014; 24(03):07-11.

44. 44. Xie T, Yang Z, Dai G, Yan K, Tian Y, Zhao Det al. Evaluation of the oral health status in Chinese hemodialysis patients. Hemodial Int, 2014; 18(3):668-73.

45. 45. Monsarrat P, Blaizot A, Kémoun P, Ravaud P, Nabet C, Sixou M, et al. Clinical research activity in periodontal medicine: a systematic mapping of trial registers. J Clin Periodontol, 2016; 43(5):390-400. 
46. 46. Spezzia S, Calvoso Jr. R. Proteína C reativa, aterosclerose e doenças periodontais. Braz J Surg Clinic Res 2013;4(1):63-7.

47. 47. Chambrone L, Foz AM, Guglielmetti MR, Pannuti CM, Artese HP, Feres M, et al. Periodontitis and chronic kidney disease: a systematic review of the association of diseases and the effect of periodontal treatment on estimated glomerular filtration rate. J Clin Periodontol, 2013; 40(5):443-56.

48. 48. Almeida S, Figueredo CM, Lemos C, Bregman R, Fischer RG. Periodontal treatment in patients with chronic kidney disease: a pilot study. J Periodont Res, 2016; 52(2):262-7. 